\title{
Modeling Michael Jarrell's CSP in Gelisp
}

\author{
Mauricio Toro \\ Universidad Eafit
}

October 4, 2018

\section{Introduction}

A Constraint Satisfaction Problem (CSP) is a mathematical problem where one must find objects that satisfy a number of constraints (i.e. criteria over those variables). CSP's provide a declarative way to represent combinatorial problems, specifying constraints instead of a sequence of steps to find the solution (as used in imperative programming). Additionally, it is possible to specify heuristics (rules to choose between branches during search).

CSP's in computer music are used to solve harmonic, rhythmic or melodic problems. In addition, they can be used for automatic generation of musical structures satisfying a set of rules. For instance, the CSP proposed by compositor Michael Jarrell, which we explain in this document and detail its implementation. Usually, a CSP is represented by a script defining the variables, their domain, and its constraints.

Instead of writing a script, in Gelisp for OpenMusic (OM) we represent a program with a special patch. A patch is a visual algorithm, in which boxes represent functional calls, and connections are functional compositions. Inside this CSP patch, we can place special boxes: to connect each constraint in the CSP, to define variable and value heuristics, to define a time limit in the search, to connect the list of variables that we want to observe, and a box to connect the variable to be the optimization criterion during the search.

Furthermore, we provide a variety of boxes to represent simple constraints (e.g., $a=b$ and $a<2$ ) and high-level constraints (e.g., "the motive $A$ occurs $n$ times in the sequence $S$ "). The output of a CSP patch can be connected to three different kind of boxes: to find one solution, to find all the solutions, and to perform propagation (narrow the domain of the variables) without search.

Process calculi has been applied to the modeling of interactive music systems [18, 17, 23, 28, 16, 27, 1, 24, 19, 4, 14, 11, 13, 15, 2, 22, 12, 20, 21, 10, and ecological systems [25, 6, 26, 7]. In addition, research on algorithms [5, 3, 9, and software engineering [8 also contributes to this field.

\section{Michael Jarrell's CSP}

Compositor Michael Jarrell proposed a CSP for automatic music generation. This CSP defines constraints to generate $n$ notes. The notes have two type of segmentation: for the chords and for the motives. Each note belongs to a chord or an ambitus (e.g., the interval [60..70]). In addition, there are interval motives and number of ocurrences in each segment.

Moreover, the first and the last note of the sequence are fixed (optionally). Finally, it is possible to have absolute or non-absolute intervals for the motives and allowing octaviation for the chords, the limits, or the motives. For instance, using octaviation, the pitch 62 (in MIDI format) is equivalent to 50,74 or 86 . On the other hand, using absolute intervals, the interval 4 is equal to the interval -4 . Following, we define formally the CSP for non-absolute intervals. For simplicity (in the formal description), we do not include octaviation, segmentation, nor ambitus.

\section{Inputs:}

- Motives $\left[M_{1} \ldots M_{A}\right]$ 
- Occurrences $\left[O M_{1} \ldots O M_{A}\right]$

- Chord $C$

- Limits $L_{1}, L_{2}$

Variables: $V_{1} \ldots V_{n}$

Domains: $[0 . .127] \ldots[0 . .127]$

\section{Constraints:}

- $C_{0} V_{n}=L_{2}$

- $C_{1} V_{0}=L_{1}$

- $C_{2} \forall_{1<i<n} \operatorname{Dom}\left(V_{i}\right)=C$

- $C_{3} \forall_{1<i<A} \mid\left\{M_{i}, M_{i}\right.$ is a subsequence of $\left.\left\{V_{i+1}-V_{i}, i<n\right\}\right\} \mid=O M_{i}$

A previous attempt to solve this problem used OmBacktrack. Unfortunately, that library is no longer available in current version of OM. For that reason, we decided to model this problem in Gelisp using graphical boxes.

\subsection{The boxes}

The graphical representation is composed by a few graphical boxes, without representing loops explicitly. The CSP is defined using the CSP box (fig 1). This box is used to define the constraints and the search heuristics.

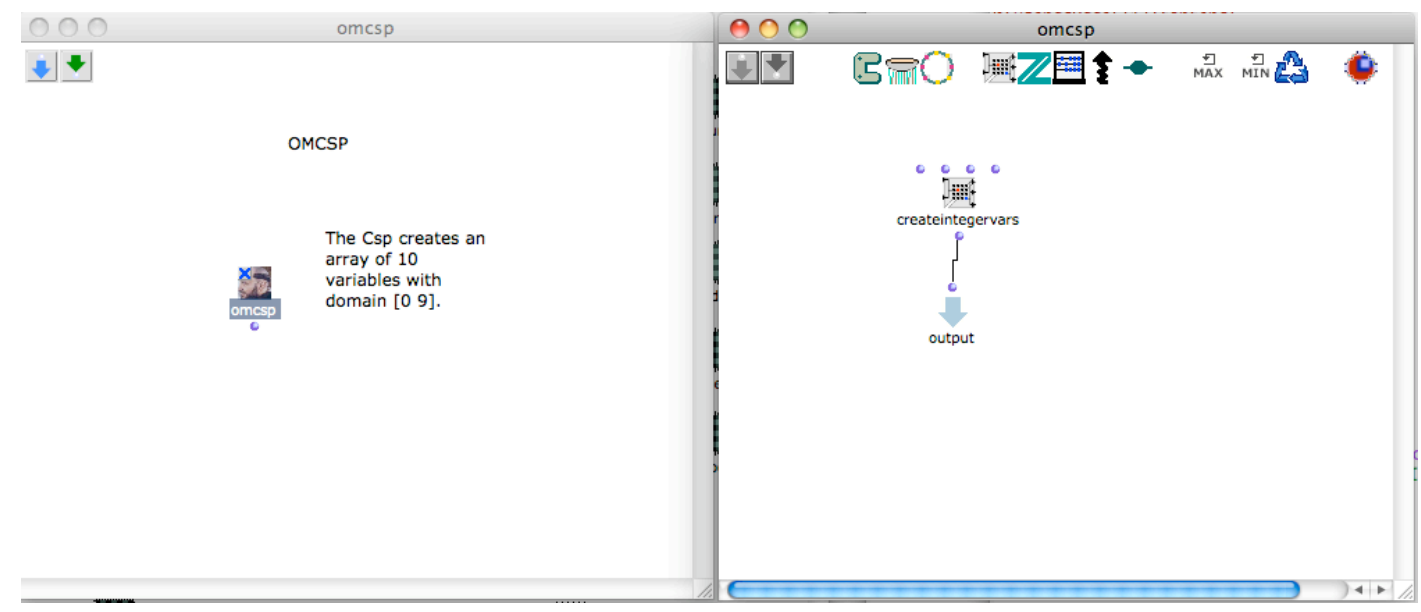

Figure 1: OMCsp Box

The limits are easily represented by an upper limit box and a lower limit box (fig. 2). Those boxes receive the list of variables, the value for the limit, and the octaviation parameter. The domain of the variables is easily determined by the member-of box (fig. 3). Using this box, we can post a constraint depending on the chord segment. In addition, it allows octaviation or ambitus (considering two notes as an ambitus or as a chord). Fig 4 presents the constraint $C_{3}$. Note how we use map iterators, the $x \rightarrow d x$, and motives-occurs= boxes to find the intervals of each motives segment and to say how many occurrences of the motives are, respectively.

\subsection{The Inputs}

0 . Motives. This is the list of motives desired for each segment.

1. Quantities. These are the quantities desired for each segment and for each motive. 


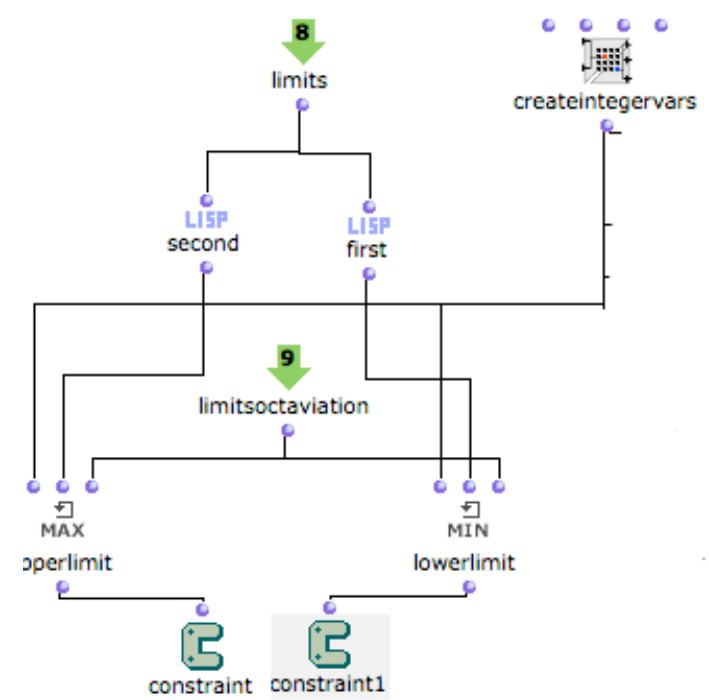

Figure 2: Constraints $C_{0}$ and $C_{1}$ of Jarrell's CSP

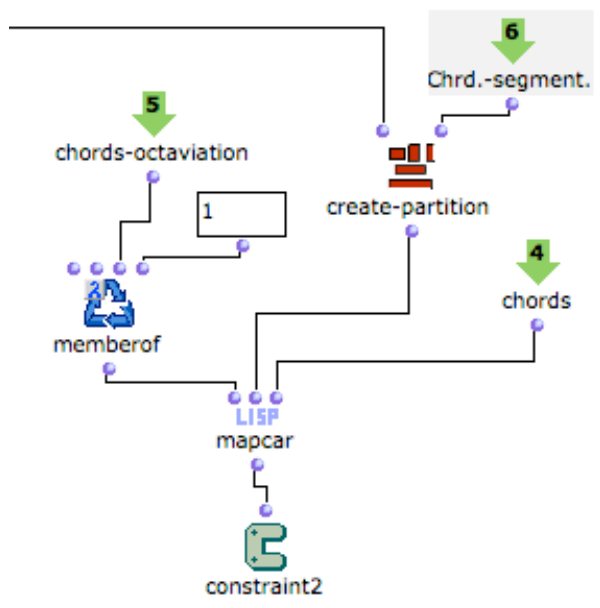

Figure 3: Constraint $C_{2}$ of Jarrell's CSP

2. Intervals type. The intervals can be absolute, non-absolute or modulo 12 .

3. Motives segmentation. This is a list with the length for each motives segment.

4. Chords. These are the chords to setup the domain of the variables.

5. Chords Octaviation. This is a parameter to allow or not octaviation of the chords.

6. Chords segmentation. This is a list with the length of each chord segment.

7. Optimization criterion: Increase. This is the optimization criterion. 1 - means the optimization criterion is the number of increasing intervals. 2 - the number of decreasing intervals. 0 - No optimization criterion.

8. Limits. This is a list with the lower and upper limits. If the list is empty, this constraints are not posted.

9. Limits octaviation. This parameter setup the octaviation for the limits. 


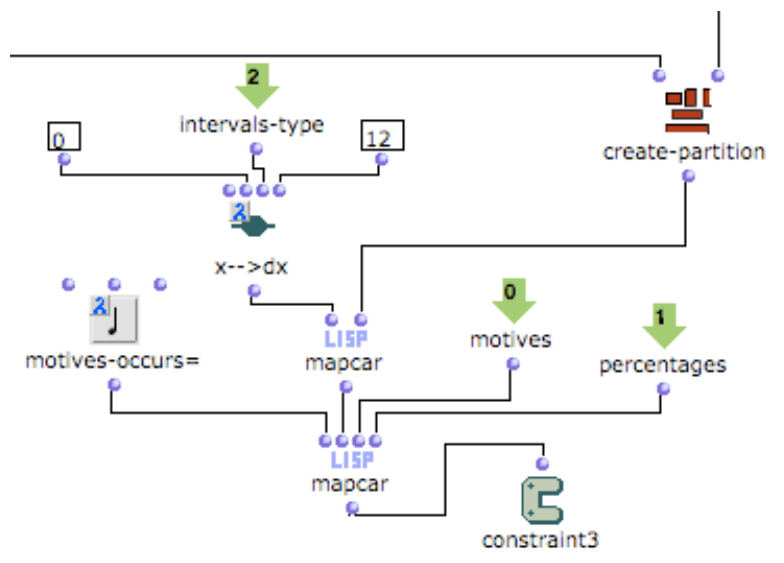

Figure 4: Constraint $C_{3}$ of Jarrell's CSP

10. Time limit. Time limit is a parameter for the maximum waiting time to find a solution.

11. Minimum value. This is the maximum value a pitch can have.

12. Maximum value. This is the minimum value a pitch can have.

\section{References}

[1] A. Allombert, M. Desainte-Catherine, and M. Toro. Modeling temporal constrains for a system of interactive score. In G. Assayag and C. Truchet, editors, Constraint Programming in Music, chapter 1, pages 1-23. Wiley, 2011.

[2] J. Aranda, G. Assayag, C. Olarte, J. A. Pérez, C. Rueda, M. Toro, and F. D. Valencia. An overview of FORCES: an INRIA project on declarative formalisms for emergent systems. In P. M. Hill and D. S. Warren, editors, Logic Programming, 25th International Conference, ICLP 2009, Pasadena, CA, USA, July 14-17, 2009. Proceedings, volume 5649 of Lecture Notes in Computer Science, pages 509-513. Springer, 2009.

[3] J. D. A. Moreno, S. Passos, and M. Toro. On-line assembling mitochondrial DNA from de novo transcriptome. CoRR, abs/1706.02828, 2017.

[4] C. Olarte, C. Rueda, G. Sarria, M. Toro, and F. Valencia. Concurrent Constraints Models of Music Interaction. In G. Assayag and C. Truchet, editors, Constraint Programming in Music, chapter 6, pages 133-153. Wiley, Hoboken, NJ, USA., 2011.

[5] C. Patiño-Forero, M. Agudelo-Toro, and M. Toro. Planning system for deliveries in Medellín. ArXiv e-prints, Nov. 2016.

[6] A. Philippou and M. Toro. Process Ordering in a Process Calculus for Spatially-Explicit Ecological Models. In Proceedings of MOKMASD'13, LNCS 8368, pages 345-361. Springer, 2013.

[7] A. Philippou, M. Toro, and M. Antonaki. Simulation and Verification for a Process Calculus for Spatially-Explicit Ecological Models. Scientific Annals of Computer Science, 23(1):119 $167,2013$.

[8] M. Raul, T. Mauricio, and C. Luz. Definicion de la arquitectura de referencia de un dominio: de la elucidacion al modelado. In R. Mazo, editor, Guia para la adopcion industrial de lineas de productos de software, pages 193-210. Editorial Eafit, 2018.

[9] J. M. C. Restrepo, A. F. Z. Palacio, and M. Toro. Assembling sequences of DNA using an on-line algorithm based on debruijn graphs. CoRR, abs/1705.05105, 2017. 
[10] M. Toro. Exploring the possibilities and limitations of concurrent programming for multimedia interaction and graphical representations to solve musical csp's. Technical Report 2008-3, Ircam, Paris.(FRANCE), 2008.

[11] M. Toro. Probabilistic Extension to the Factor Oracle Model for Music Improvisation. Master's thesis, Pontificia Universidad Javeriana Cali, Colombia, 2009.

[12] M. Toro. Towards a correct and efficient implementation of simulation and verification tools for probabilistic ntcc. Technical report, Pontificia Universidad Javeriana, May 2009.

[13] M. Toro. Structured interactive musical scores. In M. V. Hermenegildo and T. Schaub, editors, Technical Communications of the 26th International Conference on Logic Programming, ICLP 2010, July 16-19, 2010, Edinburgh, Scotland, UK, volume 7 of LIPIcs, pages 300-302. Schloss Dagstuhl - Leibniz-Zentrum fuer Informatik, 2010.

[14] M. Toro. Structured Interactive Scores: From a simple structural description of a multimedia scenario to a real-time capable implementation with formal semantics. $\mathrm{PhD}$ thesis, Univeristé de Bordeaux 1, France, 2012.

[15] M. Toro. Structured interactive music scores. CoRR, abs/1508.05559, 2015.

[16] M. Toro. Probabilistic Extension to the Concurrent Constraint Factor Oracle Model for Music Improvisation. ArXiv e-prints, Feb. 2016.

[17] M. Toro. Probabilistic Extension to the Concurrent Constraint Factor Oracle Model for Music Improvisation . Inteligencia Artificial, 57(19):37-73, 2016.

[18] M. Toro. CURRENT TRENDS AND FUTURE RESEARCH DIRECTIONS FOR INTERACTIVE MUSIC. Journal of Theoretical and Applied Information Technology, 69(16):5569-5606, 2018.

[19] M. Toro, C. Agón, G. Assayag, and C. Rueda. Ntccrt: A concurrent constraint framework for real-time interaction. In Proc. of ICMC '09, Montreal, Canada, 2009.

[20] M. Toro and M. Desainte-Catherine. Concurrent constraint conditional branching interactive scores. In Proc. of SMC '10, Barcelona, Spain, 2010.

[21] M. Toro, M. Desainte-Catherine, and P. Baltazar. A model for interactive scores with temporal constraints and conditional branching. In Proc. of Journées d'Informatique Musical (JIM) '10, May 2010.

[22] M. Toro, M. Desainte-Catherine, and J. Castet. An extension of interactive scores for multimedia scenarios with temporal relations for micro and macro controls. In Proc. of Sound and Music Computing (SMC) '12, Copenhagen, Denmark, July 2012.

[23] M. TORO, M. DESAINTE-CATHERINE, and J. CASTET. An extension of interactive scores for multimedia scenarios with temporal relations for micro and macro controls. European Journal of Scientific Research, 137(4):396-409, 2016.

[24] M. Toro, M. Desainte-Catherine, and C. Rueda. Formal semantics for interactive music scores: a framework to design, specify properties and execute interactive scenarios. Journal of Mathematics and Music, 8(1):93-112, 2014.

[25] M. Toro, A. Philippou, S. Arboleda, M. Puerta, and C. M. Vélez S. Mean-field semantics for a process calculus for spatially-explicit ecological models. In C. A. Muñoz and J. A. Pérez, editors, Proceedings of the Eleventh International Workshop on Developments in Computational Models, Cali, Colombia, October 28, 2015, volume 204 of Electronic Proceedings in Theoretical Computer Science, pages 79-94. Open Publishing Association, 2016. 
[26] M. Toro, A. Philippou, C. Kassara, and S. Sfenthourakis. Synchronous parallel composition in a process calculus for ecological models. In G. Ciobanu and D. Méry, editors, Proceedings of the 11th International Colloquium on Theoretical Aspects of Computing - ICTAC 2014, Bucharest, Romania, September 17-19, volume 8687 of Lecture Notes in Computer Science, pages 424-441. Springer, 2014.

[27] M. TORO, C. RUEDA, C. AGÓN, and G. ASSAYAG. Ntccrt: A concurrent constraint framework for soft real-time music interaction. Journal of Theoretical $\&$ Applied Information Technology, 82(1), 2015.

[28] M. TORO, C. RUEDA, C. AGÓN, and G. ASSAYAG. Gelisp: A framework to represent musical constraint satisfaction problems and search strategies. Journal of Theoretical 83 Applied Information Technology, 86(2), 2016. 